\title{
Risk factors for the treatment outcome of retreated pulmonary tuberculosis patients in China: an optimized prediction model
}

\author{
X.-M. WANG ${ }^{1} \dagger$, S.-H. YIN ${ }^{1} \dagger$, J. DU ${ }^{2} \dagger$, M.-L. DU ${ }^{1 *}$, P.-Y. WANG ${ }^{3}$, J. WU ${ }^{4}$, \\ C. M. HORBINSKI ${ }^{5}$, M.-J. WU ${ }^{5}$, H.-Q. ZHENG ${ }^{1}$, X.-Q. XU ${ }^{1}$, W. SHU ${ }^{2}$ AND Y.-J. ZHANG \\ ${ }^{1}$ School of Public Health, Inner Mongolia Medical University, Hohhot 010110, China \\ ${ }^{2}$ Beijing Tuberculosis and Thoracic Tumor Research Institute, Beijing Chest Hospital, Beijing 101149, China \\ ${ }^{3}$ Department of Social Medicine and Health Education, School of Public Health, Peking University, Beijing \\ 100191, China \\ ${ }^{4}$ Division for NCD Control and Prevention and Community Health, Center for Disease Control and Prevention in \\ China, Beijing 102200, China \\ ${ }^{5}$ Feinberg School of Medicine, Northwestern University, Chicago, IL 60611, USA \\ ${ }^{6}$ College of Traditional Chinese Medicine, Inner Mongolia Medical University, Hohhot 010110, China
}

Received 7 September 2016; Final revision 28 February 2017; Accepted 6 March 2017;

first published online 11 April 2017

\section{SUMMARY}

Retreatment of tuberculosis (TB) often fails in China, yet the risk factors associated with the failure remain unclear. To identify risk factors for the treatment failure of retreated pulmonary tuberculosis (PTB) patients, we analyzed the data of 395 retreated PTB patients who received retreatment between July 2009 and July 2011 in China. PTB patients were categorized into 'success' and 'failure' groups by their treatment outcome. Univariable and multivariable logistic regression were used to evaluate the association between treatment outcome and sociodemographic as well as clinical factors. We also created an optimized risk score model to evaluate the predictive values of these risk factors on treatment failure. Of 395 patients, 99 $(25 \cdot 1 \%)$ were diagnosed as retreatment failure. Our results showed that risk factors associated with treatment failure included drug resistance, low education level, low body mass index $(<18 \cdot 5)$, long duration of previous treatment ( $>6$ months), standard treatment regimen, retreatment type, positive culture result after 2 months of treatment, and the place where the first medicine was taken. An Optimized Framingham risk model was then used to calculate the risk scores of these factors. Place where first medicine was taken (temporary living places) received a score of 6 , which was highest among all the factors. The predicted probability of treatment failure increases as risk score increases. Ten out of 359 patients had a risk score $>9$, which corresponded to an estimated probability of treatment failure $>70 \%$. In conclusion, we have identified multiple clinical and socio-demographic factors that are associated with treatment failure of retreated PTB patients. We also created an optimized risk score model that was effective in predicting the retreatment failure. These results provide novel insights for the prognosis and improvement of treatment for retreated PTB patients.

Key words: Pulmonary tuberculosis, retreatment, risk factors, risk score.

\footnotetext{
* Author for correspondence: Maolin Du, Inner Mongolia Medical University, Hohhot, China.

(Email: dumaolin2016@163.com)

$\uparrow$ These authors contributed equally to this work.
} 


\section{INTRODUCTION}

Tuberculosis (TB) remains a critical health issue worldwide, particularly in developing countries. According to the World Health Organization (WHO) [1], 6.31 million TB cases were reported in 2014, including 6.05 million new cases and 260000 retreated patients. Moreover, TB is also one of the top 10 causes of death in the world, with 1.5 million deaths from the disease in 2014. Despite great efforts in prevention and treatment, China still has the third largest population of TB patients in the world. In 2014, 94000 new TB cases and 40000-50000 retreated TB cases were reported in China [1]. Meanwhile, there are several new challenges in the battle against $\mathrm{TB}$, including the spread of multi-drug-resistant tuberculosis (MDR-TB) and co-infection of HIV and TB. WHO is making great efforts to control TB, as exemplified by the MDGs (Millennium Development Goals), Stop TB Strategy, SDGs (Sustainable Development Goals), and the End TB Strategy [2-4]. The most recent five TB epidemiological surveys in China showed that, among total TB cases, the frequency of retreated cases were $45 \cdot 6 \%, 48 \cdot 5 \%, 38 \cdot 9 \%, 26 \cdot 1 \%$, and $11 \cdot 8 \%$, respectively [5]. Although the frequency of retreated TB cases is decreasing, there are still a big number of retreated TB cases. TB patients with retreatment failure are usually considered as MDR-TB, and thus receive complicated and expensive treatment [6].Many factors have been reported to be associated with the treatment failure of retreated TB patients, including biological factors, susceptibility test and socio-economic factors, such as bacillus Calmette-Guérin (BCG) vaccination, drug susceptibility test, occupation, education level, and compliance [7-11]. However, their exact roles and values in predicting the treatment failure remain unclear. As pulmonary tuberculosis (PTB) patients account for about $95 \%$ of the TB cases, in this study, we aimed to identify the risk factors for treatment failure in retreated PTB patients, using an optimized model to assess their values in predicting treatment failure.

\section{METHODS}

\section{Study participants and definition of index}

\section{Study participants}

Participants of this study included PTB patients who received retreatment between July 2009 and July 2011 from one of the 24 hospitals or TB prevention and control institution in Beijing, Harbin, Inner Mongolia, Guangdong, Zhejiang, and other provinces in China.

\section{Inclusion criteria.}

(1) Retreated TB patients with clear treatment outcome in prior treatment,

(2) Sputum smear positive,

(3) No serious complication,

(4) Voluntarily participated in the study and signed the informed consent form.

\section{Exclusion criteria.}

(1) Nontuberculous mycobacteria (NTM) lung disease,

(2) Negative in smear test,

(3) MDR-TB or extensively drug-resistant tuberculosis (XDR-TB) as suggested by drug susceptibility test,

(4) Allergic to any kind of anti-TB drugs.

\section{Definition of index}

Retreatment type. According to National TB control program implementation guide in China (version 2009), retreated patients was categorized into three types:

(1) Relapse: A PTB patient was declared cured or treatment completed by a physician at the end of their most recent course of treatment, but is now found to be sputum smear positive;

(2) Initial treatment failure: A PTB patient is found to be sputum smear positive at month 5 or later during treatment;

(3) Others: when a PTB patient cannot be defined as 'relapse' or 'initial treatment failure', including the one who returns, immigrates, or irregularly and irrationally uses anti-TB drugs over 1 month.

Treatment outcome. The treatment outcome was categorized according to National TB control program implementation guide in China (version 2008). These categories include:

(1) Cure: A smear-positive PTB patient who completes prescribed medication and has two consecutive negative smear results in the last month of the treatment;

(2) Treatment completion: A smear-positive PTB patient who completes prescribed medication with negative smear result at the most recent 
check-up, but no result available at the end of treatment;

(3) Treatment success: A PTB patient who completes the treatment and is cured;

(4) Treatment failure: A PTB patient whose sputum smear is positive at month 5 or later during treatment, or a smear-negative PTB patient who becomes smear positive during treatment;

(5) Death: includes TB-specific death and non-TBspecific death.

- TB-specific death: A PTB patient who dies from disease progression or complications, including hemoptysis, pneumothorax, pulmonary heart disease, systemic failure, extra-PTB etc.

- Non-TB-specific death: A PTB patient who dies

(6) from other causes rather than TB;

Lost to follow-up: A PTB patient who did not start treatment or whose treatment was interrupted for two consecutive months or more.

\section{Treatment regimen.}

(1) Standard retreatment regimen: defined as WHO recommended standard regimen for retreatment of PTB (2HREZS/6HRE and 3HRZE/6HRE);

(2) Optimal regimen: includes high-dose regimen $\left(2 \mathrm{HL}_{2} \mathrm{ZS} / 2 \mathrm{HL}_{2} \mathrm{EZS} / 4 \mathrm{HL}_{2} \mathrm{E}\right)$, long-treatment-duration regimen $\left(2 \mathrm{HL}_{2} \mathrm{EZS} / 2 \mathrm{HL}_{2} \mathrm{EZS}_{3} / 8 \mathrm{HL}_{2} \mathrm{E}\right)$ and individual regimen.

High-dose regimen: when the duration of intensive treatment is prolonged to 4 months. The dose will be given to a patient according to his/her weight, at $\mathrm{H}(0 \cdot 3) / \mathrm{d}$ for weight $<50 \mathrm{~kg}$, and $\mathrm{H}(0 \cdot 4-0 \cdot 5) / \mathrm{d}, L_{2}$ (0.6) 2 times/week for weight $\geqslant 50 \mathrm{~kg}$. Regular dose will be used for ethambutol and pyrazinamide, and the course of treatment will last 8 months.

Long-treatment-duration regimen: when the duration of intensive treatment is prolonged to 4 months. The dose will be given at $\mathrm{H}(0 \cdot 3) / \mathrm{d}, L_{2}(0 \cdot 45-0 \cdot 6) 2$ times/ week. Regular dose will be used for ethambutol and pyrazinamide, and the course of treatment will last 12 months;

Individual regimen: Drug replacement based on standard retreatment regimen according to drug resistance result of each patient.

\section{Ethics statement}

Ethical approval was obtained from Inner Mongolia Medical University and IRB of the Beijing
Tuberculosis and Thoracic Tumor Institution, Capital Medical University affiliated Beijing Chest Hospital, and the other collaboration institutions. All patients had signed informed consent prior to enrollment into the study.

\section{Statistical analysis}

All statistical analyses were performed using SPSS 19.0; significance tests were two-sided with $P \leqslant 0 \cdot 05$ considered statistically significant. Univariable logistic regression was used to compare socio-demographic characteristics, health-related behavior, previous treatment information, clinical features of diagnosis between treatment success and failure groups in retreated PTB patients, with treatment outcome as the independent variable (treatment success was assigned as 0 , treatment failure as 1). Dummy variables and variable assignment could be seen in Table 1 . The variables with $P$-values $\leqslant 0 \cdot 10$ in the univariable analysis were then included in the multivariable logistic regression model and further filtered at $P$-value $\leqslant 0 \cdot 05$. The selected variables in the final multivariable prediction model were considered as the risk factors for treatment failure. Odds ratio (OR) and the $95 \%$ confidence interval (CI) for each variable were calculated. Receiver operating characteristic (ROC) curve was then used to assess the predicted ability of the final multivariable model, determine the cut-off value of predicted probability, obtain the area under curve (AUC), and calculate sensitivity and specificity for predicted probability of retreatment failure. The diagnostic accuracy of the multivariable model was considered as non-significant when $\mathrm{AUC}<0 \cdot 5$, poor when AUC between 0.5 and 0.7 , good when AUC between 0.7 and 0.9 , and high when AUC $>0.9$. The larger the AUC, the better the predicted ability. Optimized Framingham risk model was then used to calculate risk scores based on the selected risk factors and the corresponding coefficients from the multivariable prediction model [12] (Method 1, Table S1). Risk scores were then categorized into three groups according to estimated probability of treatment failure: low $(<19 \%)$, moderate $(19 \%$ to $70 \%)$, and high $(>70 \%)$.

\section{RESULTS}

\section{Baseline characteristics of retreated PTB patients}

Among 395 retreated smear-positive PTB patients, 99 $(25 \cdot 1 \%)$ of them were in the treatment failure group 
Table 1. Variable assignment

\begin{tabular}{|c|c|}
\hline Variable & Variable assignment \\
\hline $\operatorname{BMI}\left(\mathrm{kg} / \mathrm{m}^{2}\right)$ & $0=\mathrm{BMI} \geqslant 18 \cdot 5,1=\mathrm{BMI}<18 \cdot 5$ \\
\hline Level of education & $0=$ Low (illiteracy, elementary school, or middle school), $1=$ high (college, university, or above) \\
\hline $\begin{array}{l}\text { Institution where the first } \\
\text { diagnosis was made }\end{array}$ & $\begin{array}{l}0=\mathrm{TB} \text { prevention and control institution, specialized hospital, } 1=\text { general hospital, } 2=\text { private } \\
\text { hospital or others }\end{array}$ \\
\hline $\begin{array}{l}\text { Place where first medicine } \\
\text { was taken }\end{array}$ & $\begin{array}{l}0=\text { Home, } 1=\text { medical institutions (TB prevention and control institution, TB specialized } \\
\text { hospital, or general hospital), } 2=\text { temporary living places (short-term living place, hotel or the } \\
\text { places where homeless people stay) }\end{array}$ \\
\hline Number of treatment & $0=1,1=2$ or more \\
\hline History of BCG vaccination & $0=$ No, $1=$ yes \\
\hline Previous treatment duration & $0=\leqslant 6$ months, $1=>6$ months \\
\hline Type of retreatment & $\begin{array}{l}0=\text { Relapse, } 1=\text { initial treatment failure, } 2=\text { others (patients with returning, immigration, or } \\
\text { irregular and irrational use of anti-TB drugs over } 1 \text { month) }\end{array}$ \\
\hline Treatment regimen & $0=$ Standard retreatment regimen, $1=$ optimal regimen \\
\hline Medication supervision & $0=$ No, $1=$ yes \\
\hline $\begin{array}{l}\text { Culture result after } 2 \text { months } \\
\text { of treatment }\end{array}$ & $0=$ Negative, $1=$ positive \\
\hline Drug resistance & $0=$ No, $1=$ yes \\
\hline
\end{tabular}

and $296(74.9 \%)$ were treatment success group. The median age of all the patients was 43 (Q1-Q3: 31·7553.00 years, minimum-maximum: $12-79$ years). The majority of patients were male, Han, low educated, married, and local residence, which accounted for $75 \cdot 9 \%, 94 \cdot 7 \%, 88 \cdot 4 \%, 76 \cdot 2 \%$, and $69 \cdot 6 \%$, respectively. The $63.5 \%$ of them were relapsed PTB. Patients in the failure group were younger than those in success group. Patients with drug resistance had a higher treatment failure rate than those sensitive with drug. The univariable logistic regression identified 15 variables statistically associated with higher treatment failure rate. Those variables included low body mass index (BMI < 18.5), low education level, first diagnosed at private hospital, living alone, no history of BCG vaccination, long duration of previous treatment ( $>6$ months), other retreatment type rather than relapse or initial treatment failure, receiving more than two episodes of treatment, high symptom scores before treatment $(\geqslant 5)$, having medical supervision this time, receiving standard treatment regimen, having positive culture result after 2 months of treatment. Patients who took first medicine at home had the lowest treatment failure rate, compared with those at medical institutions (TB prevention and control institution, TB specialized hospital, or general hospital), or temporary living places, other retreatment type rather than relapse or initial treatment failure (defined as patients who return, immigrate, or irregularly and irrationally use of anti-TB drugs over 1 month), (Tables 2 and 3 could be seen in the final document).

\section{Risk factors associated with treatment failure among retreated PTB patients}

A multivariable logistic regression model was conducted, including the 15 variables significantly associated with treatment outcome in the univariable logistic regression. The results indicated that patients were at a higher risk of treatment failure when they were having drug resistance, receiving standard retreatment regimen or positive culture result after 2 months. Moreover, patients were more likely to experience treatment failure if they had low education level, low BMI $(<18 \cdot 5)$, or long duration of previous treatment duration ( $>6$ months). Patients who took first medicine at home had a lower risk of failure, compared with those at temporary living places other than home or medical institutions. Moreover, retreatment type was also a risk factor for treatment failure (Table 3). The sensitivity, specificity, and positive predictive value of the multivariable prediction model were $75 \cdot 0 \%, 65 \cdot 0 \%$, and $38.4 \%$, respectively (Table 4). The predictive ability (as assessed by AUC) of the multivariable prediction model was 0.774 (95\% CI 0.715-0.883) (Fig. 1).

\section{Risk score for predicting treatment failure in retreated PTB patients with complete data}

In total, 359 patients (91\%) had complete data for all selected predictors from the multivariable prediction model. There were no significant differences between all the patients and those with complete data in all 
Table 2. Baseline characteristics in retreatment PTB patients with different treatment outcomes

\begin{tabular}{|c|c|c|c|}
\hline Characteristics & $\begin{array}{l}\text { Study population } \\
(n=395)\end{array}$ & $\begin{array}{l}\text { Treatment } \\
\text { failure }(n=99)\end{array}$ & $\begin{array}{l}\text { Treatment } \\
\text { success }(n=296)\end{array}$ \\
\hline Age (years) & $43(31 \cdot 75-53 \cdot 00)$ & $41(31 \cdot 00-52 \cdot 00)$ & $46(37 \cdot 00-54 \cdot 00)$ \\
\hline \multicolumn{4}{|l|}{ Gender } \\
\hline Male & $300(75 \cdot 9)$ & $76(25 \cdot 3)$ & $224(74 \cdot 7)$ \\
\hline Female & $95(24 \cdot 1)$ & $23(24 \cdot 2)$ & $72(75 \cdot 8))$ \\
\hline \multicolumn{4}{|l|}{ Drug susceptibility } \\
\hline Drug resistance & $185(48 \cdot 8)$ & $58(31 \cdot 4)$ & $127(68 \cdot 6)$ \\
\hline Drug sensitivity & $194(51 \cdot 2)$ & $35(18 \cdot 0)$ & $159(82 \cdot 0)$ \\
\hline \multicolumn{4}{|l|}{ BMI $\left(\mathrm{kg} / \mathrm{m}^{2}\right)$} \\
\hline$<18.5$ & $105(26 \cdot 9)$ & $34(32 \cdot 4)$ & $71(67 \cdot 6)$ \\
\hline$\geqslant 18 \cdot 5$ & $286(73 \cdot 1)$ & $64(22 \cdot 4)$ & $222(77 \cdot 6)$ \\
\hline \multicolumn{4}{|l|}{ Household register } \\
\hline Local residence & $275(69 \cdot 6)$ & $66(24 \cdot 0)$ & $209(76 \cdot 0)$ \\
\hline Non-local residence & $120(30 \cdot 4)$ & $33(27 \cdot 5)$ & $87(72 \cdot 5)$ \\
\hline \multicolumn{4}{|l|}{ Ethnicity } \\
\hline Han & $374(94 \cdot 7)$ & $94(25 \cdot 1)$ & $280(74 \cdot 9)$ \\
\hline Minorities & $21(5 \cdot 3)$ & $5(23 \cdot 8)$ & $16(76 \cdot 2)$ \\
\hline \multicolumn{4}{|l|}{ Level of education } \\
\hline Low & $349(88 \cdot 4)$ & $96(27 \cdot 5)$ & $253(72 \cdot 5)$ \\
\hline High & $46(11 \cdot 6)$ & $3(6 \cdot 5)$ & $43(93 \cdot 5)$ \\
\hline \multicolumn{4}{|l|}{ Marital status } \\
\hline Single & $77(19 \cdot 5)$ & $16(20 \cdot 8)$ & $61(79 \cdot 2)$ \\
\hline Married & $301(76 \cdot 2)$ & $79(26 \cdot 2)$ & $222(73 \cdot 8)$ \\
\hline Others & $17(4 \cdot 3)$ & $4(23 \cdot 5)$ & $13(76 \cdot 5)$ \\
\hline \multicolumn{4}{|l|}{ Smoking status } \\
\hline No smoking & $168(42 \cdot 9)$ & $37(22 \cdot 0)$ & $131(78 \cdot 0)$ \\
\hline Former smoking & $167(42 \cdot 6)$ & $44(26 \cdot 3)$ & $123(73 \cdot 7)$ \\
\hline Current smoking & $57(14 \cdot 5)$ & $17(29 \cdot 8)$ & $40(70 \cdot 2)$ \\
\hline \multicolumn{4}{|l|}{ Alcohol use } \\
\hline No drinking & $216(55 \cdot 2)$ & $48(22 \cdot 2)$ & $168(77 \cdot 8)$ \\
\hline Former drinking & $154(39 \cdot 4)$ & $41(26 \cdot 6)$ & $113(73 \cdot 4)$ \\
\hline Current drinking & $21(5 \cdot 4)$ & $9(42 \cdot 9)$ & $12(57 \cdot 1)$ \\
\hline \multicolumn{4}{|l|}{ History of $B C G$ vaccination } \\
\hline Yes & $196(49 \cdot 7)$ & $40(20 \cdot 4)$ & $156(79 \cdot 6)$ \\
\hline No & $198(50 \cdot 3)$ & $59(29 \cdot 8)$ & $139(70 \cdot 2)$ \\
\hline \multicolumn{4}{|l|}{ Previous treatment duration } \\
\hline$\leqslant 6$ months & $232(59 \cdot 2)$ & $51(22 \cdot 0)$ & $181(78 \cdot 0)$ \\
\hline$>6$ months & $160(40 \cdot 8)$ & $48(30 \cdot 0)$ & $112(70 \cdot 0)$ \\
\hline \multicolumn{4}{|l|}{ Number of treatment } \\
\hline 1 & $349(88 \cdot 4)$ & $82(23 \cdot 5)$ & $267(76 \cdot 5)$ \\
\hline$\geqslant 2$ & $46(11 \cdot 6)$ & $17(37 \cdot 0)$ & $29(63 \cdot 0)$ \\
\hline \multicolumn{4}{|l|}{ Retreatment type } \\
\hline Relapse & $251(63 \cdot 5)$ & $58(23 \cdot 1)$ & $193(76 \cdot 9)$ \\
\hline Initial treatment failure & $67(17 \cdot 0)$ & $13(19 \cdot 4)$ & $54(80 \cdot 6)$ \\
\hline Others & $77(19 \cdot 5)$ & $28(36 \cdot 4)$ & $49(63 \cdot 6)$ \\
\hline \multicolumn{4}{|l|}{ Complications } \\
\hline Yes & $93(24 \cdot 0)$ & $26(28 \cdot 0)$ & $67(72 \cdot 0)$ \\
\hline No & $294(76 \cdot 0)$ & $72(24 \cdot 5)$ & $222(75 \cdot 5)$ \\
\hline \multicolumn{4}{|l|}{ Adverse reaction this time } \\
\hline Yes & $95(24 \cdot 1)$ & $29(30 \cdot 5)$ & $66(69 \cdot 5)$ \\
\hline No & $300(75 \cdot 9)$ & $70(23 \cdot 3)$ & $230(76 \cdot 7)$ \\
\hline \multicolumn{4}{|l|}{ Treatment regimen } \\
\hline Standard regimen & $110(27 \cdot 8)$ & $42(38 \cdot 2)$ & $68(61 \cdot 8)$ \\
\hline Optimal regimen & $285(72 \cdot 2)$ & $57(20 \cdot 0)$ & $228(80 \cdot 0)$ \\
\hline \multicolumn{4}{|c|}{ Culture result after 2 months of treatment } \\
\hline Negative & $302(79 \cdot 7)$ & $56(18 \cdot 5)$ & $246(81 \cdot 5)$ \\
\hline Positive & $77(20 \cdot 3)$ & $32(41 \cdot 6)$ & $45(58 \cdot 4)$ \\
\hline
\end{tabular}


Table 3. Univariable and multivariable analysis of clinical predictors for retreatment PTB patients with treatment failure

\begin{tabular}{|c|c|c|c|c|}
\hline Characteristics & $\begin{array}{l}\text { Univariable analysis } \\
\text { (OR 95\% CI) }\end{array}$ & $P$ & $\begin{array}{l}\text { Multivariable analysis } \\
\text { (OR 95\% CI) }\end{array}$ & $P$ \\
\hline Age (years) & $1 \cdot 023(1 \cdot 005-1 \cdot 041)$ & $0 \cdot 013^{*}$ & & \\
\hline Gender (female) & $1 \cdot 062(0 \cdot 621-1 \cdot 817)$ & $0 \cdot 826$ & & \\
\hline Drug susceptibility (no) & $2 \cdot 075(1 \cdot 284-3 \cdot 353)$ & $0.003^{*}$ & $2 \cdot 060(1 \cdot 165-3 \cdot 643)$ & $0 \cdot 013 *$ \\
\hline $\mathrm{BMI}\left(\geqslant 18 \cdot 5 \mathrm{~kg} / \mathrm{m}^{2}\right)$ & $1 \cdot 6611 \cdot 013-2 \cdot 723)$ & $0 \cdot 044^{*}$ & $2 \cdot 150(1 \cdot 138-4 \cdot 061)$ & $0 \cdot 018^{*}$ \\
\hline Household register (local residence) & $1 \cdot 201(0 \cdot 738-1 \cdot 955)$ & $0 \cdot 461$ & & \\
\hline Ethnicity (Han) & $0 \cdot 931(0 \cdot 332-2 \cdot 610)$ & $0 \cdot 892$ & & \\
\hline Level of education (high) & $5 \cdot 439(1 \cdot 648-17 \cdot 945)$ & $0 \cdot 005^{*}$ & $3 \cdot 732(1 \cdot 043-13 \cdot 356)$ & $0 \cdot 043 *$ \\
\hline \multicolumn{5}{|l|}{ Marital status (single) } \\
\hline Married & $1 \cdot 357(0 \cdot 739-2 \cdot 491)$ & $0 \cdot 325$ & & \\
\hline Others & $1 \cdot 173(0 \cdot 337-4 \cdot 089)$ & $0 \cdot 802$ & & \\
\hline \multicolumn{5}{|l|}{ Marital status (no smoking) } \\
\hline Former smoking & $1 \cdot 267(0 \cdot 767-2 \cdot 092)$ & $0 \cdot 356$ & & \\
\hline Current smoking & $1 \cdot 505(0 \cdot 766-2 \cdot 954)$ & $0 \cdot 235$ & & \\
\hline \multicolumn{5}{|l|}{ Alcohol use (no drinking) } \\
\hline Former drinking & $1 \cdot 270(0 \cdot 786-2 \cdot 053)$ & $0 \cdot 329$ & & \\
\hline Current drinking & $2.625(1.044-6.599)$ & $0 \cdot 040$ & & \\
\hline Paid by themselves (yes) & $0 \cdot 860(0 \cdot 531-1 \cdot 391)$ & $0 \cdot 538$ & & \\
\hline \multicolumn{5}{|l|}{$\begin{array}{l}\text { Institution where the first diagnosis was } \\
\text { made (TB-specialized hospital) }\end{array}$} \\
\hline General hospital & $0 \cdot 814(0 \cdot 469-1 \cdot 414)$ & $0 \cdot 466$ & & \\
\hline Private hospital & $4 \cdot 479(1 \cdot 229-16 \cdot 321)$ & $0 \cdot 023$ & & \\
\hline Living alone (no) & $2 \cdot 307(0 \cdot 899-5 \cdot 919)$ & $0 \cdot 082$ & & \\
\hline History of BCG vaccination (yes) & $1 \cdot 655(1 \cdot 043-2 \cdot 627)$ & $0 \cdot 032 *$ & & \\
\hline Previous treatment duration ( $\leqslant 6$ months) & $1 \cdot 521(0 \cdot 961-2 \cdot 407)$ & $0 \cdot 073$ & $1 \cdot 984(1 \cdot 109-3 \cdot 551)$ & $0 \cdot 021 *$ \\
\hline Number of treatment (1) & $1.909(0 \cdot 999-3 \cdot 648)$ & $0 \cdot 050^{*}$ & & \\
\hline \multicolumn{5}{|l|}{ History of allergies (no) } \\
\hline Yes & $0 \cdot 589(0 \cdot 167-2 \cdot 081)$ & $0 \cdot 411$ & & \\
\hline Unknown & $1 \cdot 263(0 \cdot 320-4 \cdot 983)$ & 0.739 & & \\
\hline Previous adverse reaction (no) & $1 \cdot 166(0 \cdot 623-2 \cdot 182)$ & $0 \cdot 631$ & & \\
\hline \multicolumn{5}{|l|}{ Initial diagnosed type (invasive) } \\
\hline Fibrocavitary & $1 \cdot 147(0 \cdot 589-2 \cdot 235)$ & $0 \cdot 686$ & & \\
\hline Others & $0 \cdot 562(0 \cdot 187-1 \cdot 686)$ & $0 \cdot 304$ & & \\
\hline Regularly taking medicine at first diagnosis (yes) & $1 \cdot 210(0 \cdot 740-1 \cdot 981)$ & $0 \cdot 447$ & & \\
\hline Medication supervision at first diagnosis(yes) & $1 \cdot 279(0 \cdot 779-2 \cdot 100)$ & $0 \cdot 330$ & & \\
\hline \multicolumn{5}{|l|}{ Place where first medicine was taken (home) } \\
\hline Medical institutions & $1 \cdot 615(0 \cdot 912-2 \cdot 859)$ & $0 \cdot 100$ & $2 \cdot 011(0 \cdot 950-4 \cdot 257)$ & $0 \cdot 068$ \\
\hline Temporary living places & $5 \cdot 176(1 \cdot 421-18 \cdot 849)$ & $0 \cdot 013$ & $9 \cdot 329(1 \cdot 716-50 \cdot 722)$ & $0 \cdot 010^{*}$ \\
\hline \multicolumn{5}{|l|}{ Retreatment type (relapse) } \\
\hline Initial treatment failure & $0 \cdot 801(0 \cdot 409-1 \cdot 570)$ & $0 \cdot 518$ & $0.695(0 \cdot 305-1 \cdot 584)$ & $0 \cdot 387$ \\
\hline Others & $1 \cdot 901(1 \cdot 098-3 \cdot 293)$ & $0 \cdot 022$ & $2 \cdot 081(1 \cdot 057-4 \cdot 096)$ & $0 \cdot 034 *$ \\
\hline Symptom scores before treatment $(<5)$ & $1 \cdot 634(1 \cdot 031-2 \cdot 589)$ & $0 \cdot 037^{*}$ & & \\
\hline Fibrocavitary & $1 \cdot 478(0 \cdot 911-2 \cdot 398)$ & $0 \cdot 113$ & & \\
\hline Others & $0 \cdot 719(0 \cdot 200-2 \cdot 587)$ & $0 \cdot 614$ & & \\
\hline Complications (no) & $1 \cdot 197(0 \cdot 708-2 \cdot 023)$ & $0 \cdot 503$ & & \\
\hline Adverse reaction this time (no) & $1 \cdot 444(0 \cdot 865-2 \cdot 409)$ & $0 \cdot 160$ & & \\
\hline Hospitalization (no) & $0 \cdot 854(0 \cdot 538-1 \cdot 356)$ & $0 \cdot 503$ & & \\
\hline \multicolumn{5}{|l|}{ DOT distance $(<1 \mathrm{~km})$} \\
\hline $1 \sim 5 \mathrm{~km}$ & $0 \cdot 643(0 \cdot 257-1 \cdot 612)$ & $0 \cdot 347$ & & \\
\hline $6 \sim 10 \mathrm{~km}$ & $1 \cdot 117(0 \cdot 421-2 \cdot 964)$ & $0 \cdot 823$ & & \\
\hline$>10 \mathrm{~km}$ & $0.826(0 \cdot 357-1 \cdot 909)$ & 0.654 & & \\
\hline Medical supervision this time (yes) & $3 \cdot 143(1 \cdot 147-8 \cdot 612)$ & $0 \cdot 026^{*}$ & & \\
\hline Treatment regimen (optimal regimen) & $2 \cdot 471(1 \cdot 526-4 \cdot 000)$ & $<0 \cdot 001^{*}$ & $3 \cdot 329(1 \cdot 778-6 \cdot 234)$ & $<0.001 *$ \\
\hline $\begin{array}{l}\text { Culture result after } 2 \text { months of } \\
\text { treatment month (negative) }\end{array}$ & $3 \cdot 124(1 \cdot 824-5 \cdot 351)$ & $<0 \cdot 001 *$ & $3 \cdot 498(1 \cdot 811-6 \cdot 754)$ & $<0 \cdot 001 *$ \\
\hline
\end{tabular}

Note: $* P<0 \cdot 05$.

Variables in brackets represent control groups. 
Table 4. Performance characteristics for diagnosing retreatment failure using multivariable prediction model

\begin{tabular}{lllll}
\hline \hline Value & TPR & TNR & PPV & NPV \\
\hline Predicted probability & $75 \cdot 0 \%$ & $65 \cdot 0 \%$ & $38 \cdot 4 \%$ & $90 \cdot 4 \%$ \\
\hline
\end{tabular}

TPR, true-positive rate (sensitivity); TNR, true-negative rate (specificity); PPV, positive predictive value; NPV, negative predictive value.

the selected predictors $(P>0.05)$ (Table S2). Thus, those predictors were used to calculate the risk scores in the patients with complete data. The predicted probability of treatment failure increased with increasing risk score (Fig. 2). The risk score of each risk factor was also calculated. Patients whose first medicines were taken at the temporary living places other than home or medical institutions had the highest score of 6 (Table 5). Ten out of 359 patients had a risk score $>9$, which corresponded to a probability of treatment failure higher than $70 \%$ (Table S3).

\section{DISCUSSION}

In China, the emergence of large number of retreated cases may be due to irrational drug use, mismanagement, irregular treatment, and many other reasons [13-15].Retreated TB patients usually have severe adverse reactions and tend to have mood disorders such as depression. The failure of retreatment not only affects treatment compliance and confidence, but also influences the burden on families and caregivers. Therefore, in order to enhance the prevention and improvement of retreatment regimens, it is of great importance to identify risk factors and assess their predictive value. Here, we analyzed the data from a larger survey in China to identify predictors of treatment failure for retreated PTB patients. We used an optimized risk score model to assess the value of these factors in predicting treatment failure. Our findings add novel insights for the prognosis and treatment of retreated PTB patients in China and throughout the world.

The Optimized Framingham Heart Profile (FHP) is widely used in various fields [16], including stroke, coronary heart disease, metabolic syndrome, hypertension, TB, and other diseases [17-22]. In addition, optimized FHP was also used to predict the risk of bacteremia for TB patients with HIV, which showed that predicted probability of bacteremia for TB patients with HIV increased with increasing risk

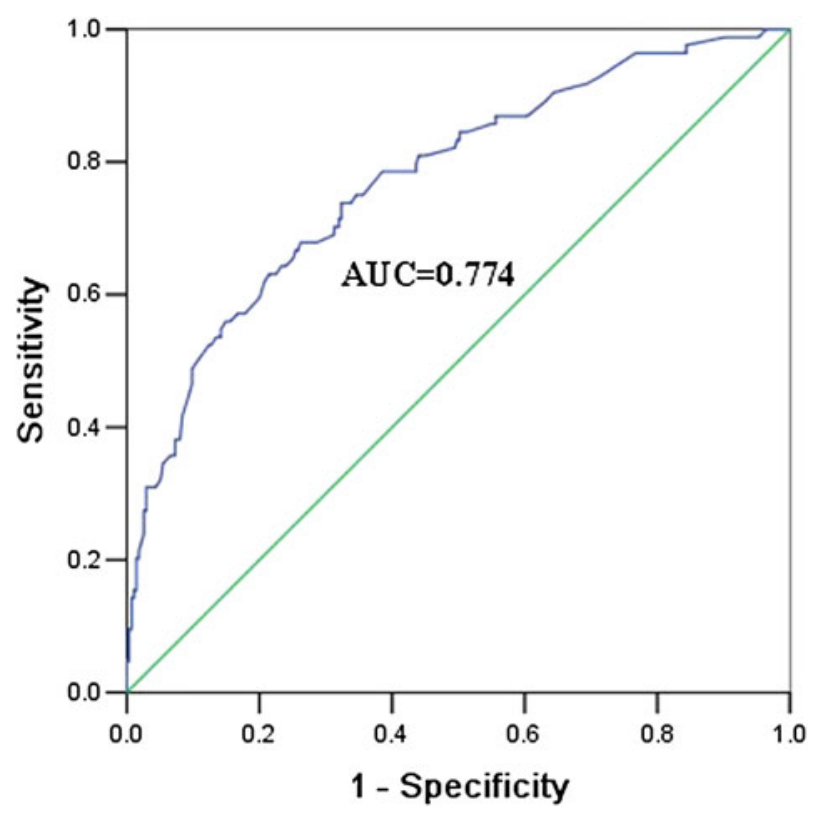

Fig. 1. ROC curves of final multivariable prediction model for retreated PTB patients with treatment failure.

score. Our results indicated that, with certain adjustments, the model could be effective in evaluating risk factors for PTB patients in China.

In this study, we found that place where first medicine was taken was an independent risk factor for the treatment failure of retreated PTB patients. Compared with patients who took medicine at home, risk score of patients who took medicine at temporary living places was much higher (6 points), which corresponded to the highest risk of treatment failure (OR 9.3293, 95\% CI 1. 716-50.722). These patients may be part of migrating populations who cannot take medicine at home, and lack the supervision of TB prevention and control institutions or community stations. In addition, our study found that patients with previous treatment duration $>6$ months had higher risk of treatment failure (OR 1.984, 95\% CI 1.109-3.551). Inappropriate treatment regimen, poor compliance and migrating may prolong treatment period. Previous study [23] had shown that prolonged initial treatment duration was a risk factor for drug resistance (OR 2-18), which could result in retreatment failure. Therefore, for these patients who take first medicine at temporary living places or have previous treatment duration $>6$ months, we should take proper interventions during their retreatment to decrease the failure rate.

The standard WHO recommended retreatment regimen (category II) for PTB is an economic and 


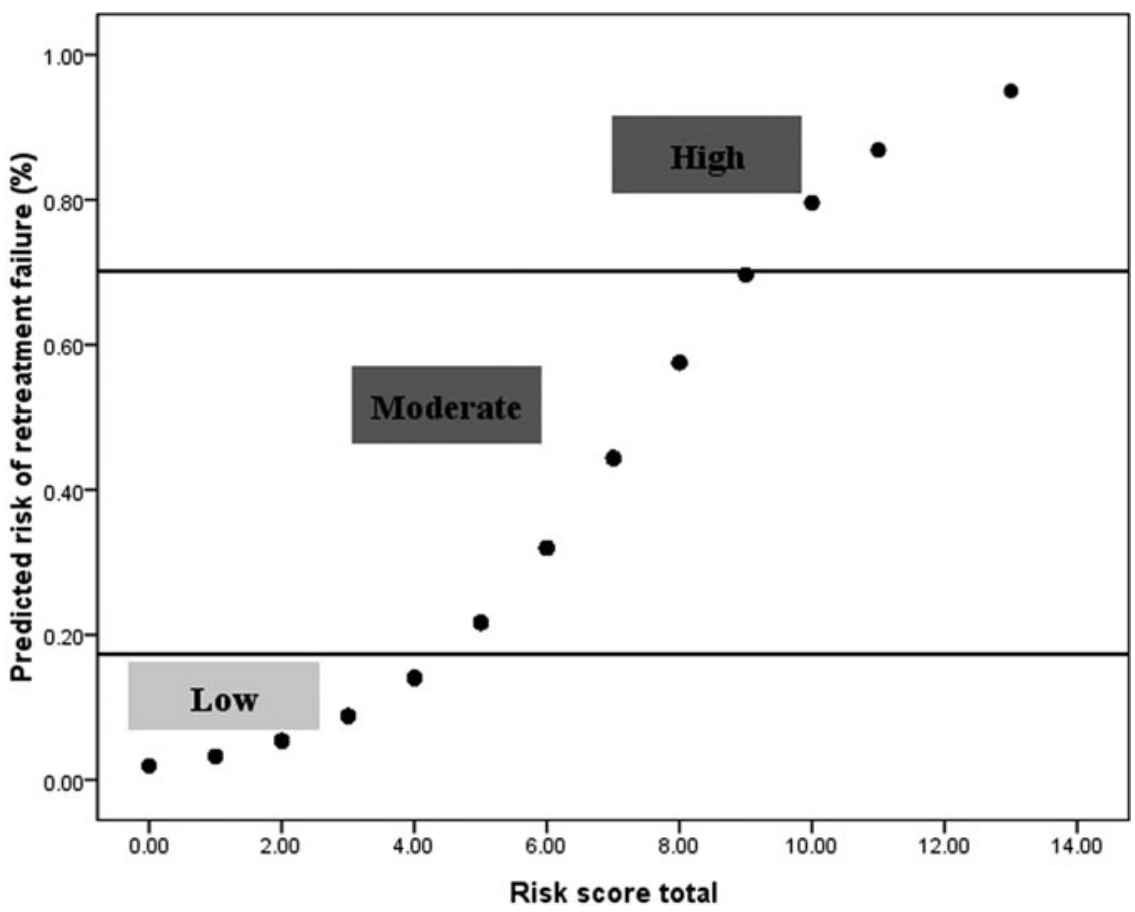

Fig. 2. Risk of retreated PTB patients with treatment failure corresponding to treatment failure risk score.

Table 5. Risk factors and the corresponding risk score assigned by multivariable logistic regression to predict retreatment failure

\begin{tabular}{|c|c|}
\hline Category & Risk score \\
\hline \multicolumn{2}{|l|}{ Level of education } \\
\hline Low & 0 \\
\hline High & 2 \\
\hline \multicolumn{2}{|l|}{ BMI $\left(\mathrm{kg} / \mathrm{m}^{2}\right)$} \\
\hline$\geqslant 18 \cdot 5$ & 0 \\
\hline$<18 \cdot 5$ & 1 \\
\hline \multicolumn{2}{|l|}{ Retreatment type } \\
\hline Relapse & 0 \\
\hline Initial treatment failure & 1 \\
\hline Others & 2 \\
\hline \multicolumn{2}{|l|}{ Treatment regimen } \\
\hline Standard regimen & 0 \\
\hline Optimal regimen & 2 \\
\hline \multicolumn{2}{|l|}{ Drug susceptibility } \\
\hline Drug resistance & 0 \\
\hline Drug sensitivity & 1 \\
\hline \multicolumn{2}{|c|}{ Place where first medicine was taken } \\
\hline Home & 0 \\
\hline Medical institutions & 1 \\
\hline Temporary living places & 6 \\
\hline \multicolumn{2}{|l|}{ Previous treatment duration } \\
\hline$\leqslant 6$ months & 0 \\
\hline$>6$ months & 1 \\
\hline \multicolumn{2}{|c|}{ Culture result after 2 months of treatment } \\
\hline Negative & 0 \\
\hline Positive & 2 \\
\hline
\end{tabular}

efficient regimen [24-26]. However, in past two decades, the collaborative researches were conducted in many countries have provided a lot of evidence that patients ultimately failed with standard retreatment regimen [27-32]. Our study showed that the risk score of standard retreatment regimen was 2 points, which indicated a relatively high risk for retreatment failure (OR 3.329, 95\% CI 1·778-6.234). This was probably because the standard retreatment regimen, comprising four kinds of first-line drug combination regimen in initial treatment plus streptomycin, was mainly proposed based on the experiences of expert. Therefore, the Standard Retreatment Regimen recommended by WHO may not be appropriate for every PTB patient. More effective treatment regimen should be developed according to the characteristics and the condition of retreated PTB patients.

Our study showed that the risk score of drug resistance was 1 point, which corresponded to a higher risk of retreatment failure than drug-sensitive TB (OR $2 \cdot 060$, 95\% CI 1.165-3.643). Consistent with these findings, a study from South Korea indicated that success rate for new patients with drug resistance $(68.8 \%)$ was much higher than that of retreated TB patients (40.7\%) [33]. Another study in China showed that the chance of treatment failure for MDR-TB was 4.7 times more than that in drug-sensitive patients [34]. 
Culture result after 2 months of treatment can be used as a comprehensive treatment effect indicator for patients after intensive treatment. Our study showed that positive culture after 2 months of treatment was an independent risk factor for treatment failure in retreated PTB patients, with a risk score of 2 points. Patients with positive culture were 2.498 times more likely to fail during treatment than those with negative results, which was consistent with the study of Zhou and co-workers [35]. Besides, our study found that the risk score of treatment failure in PTB patients with other type of retreatment was higher than patients who relapsed, with an OR of $2 \cdot 081$ (95\% CI 1.057-4.096). This might be associated with definite microbiological diagnosis in patients with relapsed PTB, while the others who return, immigrate, or irregularly and irrationally use of anti-TB drugs over 1 month were more likely to be combined with other disease, such as HIV, lung, or heart disease, which could lead to immune function impairment and increase the risk of poor treatment outcome.

Our study also found that the risk score of low education level and low BMI $(<18 \cdot 5)$ were 2 and 1 , respectively. The reason may be that lack of education and low BMI were associated with poor socioeconomic factors. Patients who had less awareness of health issues and self-care might delay in the diagnosis and treatment. Also patients with low education level will be more likely to misuse drug and discontinue drug use during treatment.

We acknowledged that this study had some limitations. First, our model may need to validate with a second cohort to strengthen our conclusions. Second, the study was performed with the data from China, thus the application of the model in other countries may require further examination. However, our study provides a good starting point for international studies on this topic, and our further study is also in progress.

\section{CONCLUSIONS}

In summary, predictors of treatment failure for retreated PTB patients included drug susceptibility, BMI, level of education, previous treatment duration, location where first medicine was taken, retreatment type, treatment regimen, and culture result after two months of treatment. The Optimized Framingham risk model built with those risk factors was proven to be effective in predicting the treatment failure, which could improve early diagnosis of treatment failure in retreated PTB patients using risk scores, especially when using standard treatment regimen. Our findings add novel insights for the prognosis and treatment of retreated PTB patients in China.

\section{SUPPLEMENTARY MATERIAL}

The supplementary material for this article can be found at https://doi.org/10.1017/S0950268817000656

\section{ACKNOWLEDGEMENTS}

This study was supported by One million projects of Science and Technology of Inner Mongolia Medical University (YKD2013KJBW006), Intelligent health monitoring and modern medical information system development based on technology of Internet of Things and National Science and Technology Major Projects (2008ZX10003-015-2).

\section{DECLARATION OF INTERESTS}

None.

\section{REFERENCES}

1. WHO. Global Tuberculosis Report 2015. Geneva: World Health Organization, 2015.

2. Jaramillo M. The Stop TB Strategy: building on and enhancing DOTS to meet the TB-related Millennium Development Goals. Geneva, Switzerland: World Health Organization [WHO], 2006.

3. MCR. The Global Plan to Stop TB, 2006-2015. International Journal of Tuberculosis \& Lung Disease the Official Journal of the International Union against Tuberculosis \& Lung Disease 2006; 10(2): 238-239.

4. WHO. Global tuberculosis control 2015. Global Tuberculosis Control 2015.

5. Lixia W, et al. Survey of the Fifth National epidemiological sampling of tuberculosis in 2010. Chinese Journal of Tuberculosis Prevention 2012; 34(08): 485 508.

6. World Health Organization. Stop TB Department Guidelines for the Programmatic Management of DrugResistant Tuberculosis. Geneva: World Health Organization, 2006.

7. Qianghong D, et al. Treatment outcomes and risk factors for retreated smear positive pulmonary tuberculosis in Wuhan City. Chinese Journal of Tuberculosis Prevention 2013; 35(10): 788-792.

8. Yingjie Y. Risk factors for the efficacy of retreated smear positive tuberculosis patients and nursing intervention. Jilin Medicine 2010; 31(34): 6350-6351.

9. Vicentin G. Evoluçäo da mortalidade por tuberculose no município do Rio de Janeiro, 1979-1995. São Paulo, Brazil: Universidade de São Paulo, 2000. 
10. Volmink J, Garner P. Systematic review of randomised controlled trials of strategies to promote adherence to tuberculosis treatment. BMJ (Clinical Research edn) 1997; 315(7120): 1403-1406.

11. Yaqin L, Yuan M, Wei C. Risk factors for compliance of retreated tuberculosis patients. Nursing Research 2011; 25(29): 2649-2651.

12. Sullivan LM, Massaro JM, D'Agostino RB. $\mathbf{S r}$ Presentation of multivariate data for clinical use: the Framingham Study risk score functions. Statistics in Medicine 2004; 23(10): 1631-1660.

13. Shiming $\mathbf{C}$, et al. Investigation and analysis on social factors of retreated smear positive pulmonary tuberculosis patients in Hunan Province. Practical Preventive Medicine 2003; 10(02): 132-134.

14. Kherosheva T, et al. Encouraging outcomes in the first year of a TB control demonstration program: Orel Oblast, Russia. The International Journal of Tuberculosis and Lung Disease: the Official Journal of the International Union against Tuberculosis and Lung Disease 2003; 7(11): 1045-1051.

15. Talay F, Kumbetli S, Altin S. Factors associated with treatment success for tuberculosis patients: a single center's experience in Turkey. Japanese Journal of Infectious Diseases 2008; 61(1): 25-30.

16. D'Agostino RB, et al. Stroke risk profile: adjustment for antihypertensive medication. The Framingham Study. Stroke; a Journal of Cerebral Circulation 1994; 25(1): 40-43.

17. Yousefzadeh G, et al. Applying the Framingham risk score for prediction of metabolic syndrome: the Kerman Coronary Artery Disease Risk Study, Iran. ARYA Atherosclerosis 2015; 11(3): 179-185.

18. Goldstein LB BC, Adams RJ, Appel LJ, Braun LT, Chaturvedi S, Creager MA, Culebras A, Eckel RH, Hart RG, Hinchey JA, Howard VJ, Jauch EC, Levine SR, Meschia JF, Moore WS, Nixon JV, Pearson TA; American Heart Association Stroke Council; Council on Cardiovascular Nursing; Council on Epidemiology and Prevention; Council for High Blood Pressure Research; Council on Peripheral Vascular Disease and Interdisciplinary Council on Quality of Care and Outcomes Research. Guidelines for the primary prevention of stroke: a guideline for healthcare professionals from the American Heart Association/American Stroke Association. Stroke 2011; 42(2): 517-584.

19. Wang H, et al. Longitudinal association of dairy consumption with the changes in blood pressure and the risk of incident hypertension: the Framingham Heart Study. The British journal of nutrition 2015; 114(11): 1887-1899.

20. Jacob ST, Pavlinac PB, Nakiyingi L, Banura P, Baeten JM, Morgan K, et al. Mycobacterium tuberculosis Bacteremia in a Cohort of HIV-infected patients hospitalized with severe sepsis in Uganda-high frequency, low clinical sand derivation of a Clinical Prediction Score. PLOS ONE 2013; 8(8): e70305.

21. Joshi PH, et al. Remnant lipoprotein cholesterol and incident coronary heart disease: the Jackson Heart and Framingham Offspring Cohort Studies. Journal of the American Heart Association 2016; 5(5).

22. Parikh NI, et al. A risk score for predicting near-term incidence of hypertension: the Framingham Heart Study. Annals of Internal Medicine 2008; 148(2): 102-110.

23. Chen $\mathbf{S}$, et al. Risk factors for multidrug resistance among previously treated patients with tuberculosis in eastern China: a case-control study. International Journal of Infectious Diseases: IJID: Official Publication of the International Society for Infectious Diseases 2013; 17(12): e1116-1120.

24. Lenjisa J. Assessment of tuberculosis retreatment case rate and its treatment outcomes at Adama Hospital Medical College, East Showa, Ethiopia. Journal of Steroids \& Hormonal Science 2015; 06(1).

25. Jones-Lopez EC, et al. Effectiveness of the standard WHO recommended retreatment regimen (category II) for tuberculosis in Kampala, Uganda: a prospective cohort study. PLoS Medicine 2011; 8(3): e1000427.

26. Mpagama SG, et al. The influence of mining and human immunodeficiency virus infection among patients admitted for retreatment of tuberculosis in Northern Tanzania. The American Journal of Tropical Medicine and Hygiene 2015; 93(2): 212-215.

27. Kritski AL, et al. Retreatment tuberculosis cases. Factors associated with drug resistance and adverse outcomes. Chest 1997; 111(5): 1162-1167.

28. Espinal MA, et al. Standard short-course chemotherapy for drug-resistant tuberculosis: treatment outcomes in 6 countries. Jama 2000; 283(19): 2537-2545.

29. He GX, et al. Follow-up of patients with multidrug resistant tuberculosis four years after standardized first-line drug treatment. PLoS ONE 2010; 5(5): e10799.

30. Green E, et al. Drug-susceptibility patterns of Mycobacterium tuberculosis in Mpumalanga province, South Africa: possible guiding design of retreatment regimen. Journal of Health, Population, and Nutrition 2010; 28(1): 7-13.

31. Mak A, et al. Influence of multidrug resistance on tuberculosis treatment outcomes with standardized regimens. American Journal of Respiratory and Critical care Medicine 2008; 178(3): 306-312.

32. Faustini A, Hall AJ, Perucci CA. Risk factors for multidrug resistant tuberculosis in Europe: a systematic review. Thorax 2006; 61(2): 158-163.

33. Choi H, et al. Predictors of pulmonary tuberculosis treatment outcomes in South Korea: a prospective cohort study, 2005-2012. BMC Infectious Diseases 2014; 14(1): 360 .

34. Jian D, et al. Reasons for failure of Standardized short term chemotherapy regimen for initial or retreated pulmonary tuberculosis patients. Chinese Journal of Parasitic Disease 2012; 7(07): 523-526.

35. Yang $\mathbf{Z}$, et al. Risk factors for sputum culture result at the end of 2nd month of treatment in MDR-TB patients. Chinese Journal of Tuberculosis Prevention 2015; 37(1): 74-79. 